\title{
BMJ Open Health insurance awareness and its uptake in India: a systematic review protocol
}

\author{
Bhageerathy Reshmi, ${ }^{1}$ Bhaskaran Unnikrishnan, ${ }^{2}$ Shradha S Parsekar (D) , ${ }^{3}$ \\ Eti Rajwar, ${ }^{3}$ Ratheebhai Vijayamma, ${ }^{4}$ Bhumika Tumkur Venkatesh (iD ${ }^{3}$
}

To cite: Reshmi B, Unnikrishnan B, Parsekar SS, et al. Health insurance awareness and its uptake in India: a systematic review protocol. BMJ Open 2021;11:e043122. doi:10.1136/ bmjopen-2020-043122

- Prepublication history for this paper is available online. To view these files, please visit the journal online (http://dx.doi. org/10.1136/bmjopen-2020043122).

Received 31 August 2020 Revised 01 March 2021 Accepted 02 March 2021

Check for updates

(C) Author(s) (or their employer(s)) 2021. Re-use permitted under CC BY-NC. No commercial re-use. See rights and permissions. Published by BMJ.

${ }^{1}$ Health Information

Management, Manipal College of Health Professionals, Manipal Academy of Higher Education, Manipal, India

${ }^{2}$ Kasturba Medical College, Mangalore, Manipal Academy of Higher Education, Manipal, India ${ }^{3}$ Public Health Evidence South Asia, Department of Health Information, Prasanna School of Public Health, Manipal Academy of Higher Education, Manipal Academy of Higher Education, Manipal, India

${ }^{4}$ Manipal Institute of

Communication, Manipal Academy of Higher Education, Manipal, India

Correspondence to Dr Bhumika Tumkur Venkatesh; bhumika.tv@manipal.edu

\section{ABSTRACT:}

Introduction Health insurance is one of the important approaches that can help in boosting universal healthcare coverage through improved healthcare utilisation and financial protection. This objectives of this review are to identify various interventions implemented in India to promote awareness of health insurance, and to provide evidence for the effectiveness of such interventions on the awareness and uptake of health insurance by the resident Indian population.

Methods and analysis A systematic review will be carried out based on the Cochrane handbook for systematic reviews of interventions. The review will include experimental and analytical observational studies that have included adult population ( $>18$ years) in India. We will include any intervention, policy or programme that directly or indirectly affects awareness or uptake of health insurance. The following outcomes will be eligible to be included: awareness or health insurance literacy, attitude such as readiness to buy health insurance or decision making, uptake of health insurance, demand-side and supply-side factors for awareness of health insurance, and awareness as a factor for uptake and re-enrolment in health insurance. Databases such as MEDLINE (PubMed), Web of Science, Scopus, 3ie impact evaluation repository and Social Science Research Network will be searched from January 2010 to 15 July 2020. Additionally, important government websites and references of the included studies will be scanned to identify potential records. Three authors, independently, will carry out screening and data extraction. Studies will be categorised into quantitative and qualitative, and mixed-methods synthesis will be employed to analyse the findings.

Ethics and dissemination This review will be based on published studies and will not recruit human participants directly, therefore, ethical clearance is not applicable. We will disseminate the final review findings in a national or international conference and publish in a peer-reviewed journal.

\section{INTRODUCTION}

Low-income and middle-income countries (LMICs) contribute to around $84 \%$ of the world population and $90 \%$ of the global burden of disease. ${ }^{1}$ People living in the LMICs rely majorly on out-of-pocket payments as the prime source for managing healthcare

\section{Strengths and limitations of this study}

This systematic review will use mixed-methods analysis involving findings from quantitative and qualitative studies conducted in India.

- We will comprehensively search the evidence in various databases, grey literature and reference and forward citations of included studies, however, the publications will be restricted to English.

- We anticipate heterogeneity owing to study designs of potentially included studies, however, to mitigate this challenge we have planned to conduct subgroup analysis based on PROGRESS-Plus framework.

expenses, that results in a massive demand for services and financial burden of households (usually catastrophic), which in turn leads to impoverishment. ${ }^{1-5}$ It is projected that every year approximately 150 million people experience financial catastrophe, by spending more than $40 \%$ on health expenses other than food. ${ }^{6}$ Families generally spend more than $10 \%$ of the household income on illness-related expenses, due to which other household expenses are affected. ${ }^{25}$ To make it worse, evidence suggests that per capita spending on healthcare in many LMICs is expected to increase in coming years. ${ }^{4}$ Additionally, the increased costs of seeking and receiving care can hinder the access to healthcare. ${ }^{7}$

The Universal Health Coverage (UHC) is embedded within the Sustainable Development Goals (SDGs) and aims 'to ensure healthy lives and promote well-being for all at all ages by $2030,{ }^{8}$ It includes financial risk protection and equal access to quality essential healthcare services. ${ }^{8} 9$ In other terms, UHC encourages equitable healthcare ${ }^{2}$ and nations across the world are committed to achieving SDGs through UHC. ${ }^{10}$

Health insurance is one of the important approaches that can help in boosting UHC through improved healthcare utilisation and 
financial protection. ${ }^{7-9} 11$ There are multiple types of insurance in LMICs that differ with providers (government vs private sector), scales and types of beneficiaries. ${ }^{8}$ However, in many LMICs, due lack of acceptability and unwillingness to pay (WTP) premiums, health insurance coverage is limited. ${ }^{24}$ This increases the risk of excluding vulnerable and at-risk population, who cannot afford to pay health insurance premium. ${ }^{8}$ Additionally, the older adults, and the individuals with disability and chronic diseases, have less probability of enrolling in health insurance schemes or their specific needs may not be covered under the scheme. ${ }^{8}$

The coverage of health insurance policies or programmes in India is improving, however, the publicly funded health insurance schemes are mostly restricted to socioeconomically backward people or government employees. ${ }^{12}$ India's first health insurance programme, launched in the 1950s, was limited to central government employees and certain low-income population. ${ }^{11}$ Over the years, the private healthcare providers' dominance in quality healthcare service provision can be seen. ${ }^{11}$ Nevertheless, many economically backward families are either deprived of healthcare or are pushed into poverty in the absence of financial protection. ${ }^{11}$ In 2002, targeted health insurance programmes for low-income households were introduced by central and state governments in partnership with private sector and non-governmental organisations (NGO). Since 2002 (recommendations of National Health Policy 2002), more than 17 health insurance schemes have been launched by various governments in India. ${ }^{11}$ The most recent one is 'Ayushman Bharat' or Pradhan Mantri Jan Arogya Yojana (PMJAY) (Prime minister's health assurance scheme) launched in 2018 to achieve UHC. PMJAY is fully financed by the government and seeks to cover 500 million citizens with an annual cover of approximately US $\$ 7000$ per household. The main aim of the PMJAY is to lessen the economic burden experienced by poor and vulnerable groups for access to healthcare facility. ${ }^{13}$

Despite the availability of multiple health insurance schemes, evidence suggests that the uptake of health insurance in India is poor. As per the recently concluded National Sample Survey Office data, there were as low as $14 \%$ rural and 18\% urban residents of India having some form of health insurance. ${ }^{14}$ The low coverage of health insurance was evident in other literature, wherein it was reported to be less than $20 \% .^{512}$ Similarly, other LMICs have reported poor registrations in the national health insurance schemes. ${ }^{891215}$

There are multiple factors that are responsible for awareness and enrolment in health insurance schemes. ${ }^{2}{ }^{4}$ These factors can be broadly divided into individual (age, gender, education, employment status, marital status), ${ }^{2916}$ and household characteristics (wealth, size of family). ${ }^{1917}$ Other factors are programme-related (premium amount, rules, regulation and procedures), social capital (trust, networks and group participation, social norms and solidarity and togetherness features of the social organisation of the community), institutional factors (regulatory mechanisms, complaint handling systems and insurance education) and supply-side factors (quality of care and distance of house from the nearest health facility). ${ }^{2}$ The aforementioned factors may also determine the consumer preference in selecting the health insurance. ${ }^{17}$ Inadequate claim returns, poor accountability and non-transparent operations hinders the uptake of health insurance. ${ }^{18}$

In Indian studies, a scant that is, 34\% of the participants who did not have health insurance were willing to pay for any health insuance. ${ }^{5}$ Previous research in LMICs suggests that financial status of household is positively associated with WTP. ${ }^{24918}$ Whereas level of education received contradictory findings, that is, a study conducted in Nigeria reported that education was negatively associated with $\mathrm{WTP}^{4}$ contrary to study conducted in Uganda, ${ }^{1}$ Ghana ${ }^{16}$ and India. ${ }^{5}$ Family who had good perceived health had less probability of getting insurance as compared with those individuals who perceived their health as poor. Similarly, those individuals who had chronic diseases were more inclined to have health insurance than those who did not have chronic diseases. ${ }^{2}$ Corruption and mistrust in the health insurance scheme ${ }^{1518}$ and expensive plans ${ }^{518}$ were some of the reasons for non-WTP. Lack of information or health insurance illiteracy is another important reason for non-WTP. ${ }^{1518}$

Health insurance literacy is defined as 'the degree to which individuals have the knowledge, ability and confidence to find and evaluate information about health plans, select the best plan for their own (or their families) financial and health circumstances, and use the plan once enrolled.' ${ }^{, 1}$ Lack of health insurance literacy or education hinders the uptake of health insurance and in many LMICs health insurance literacy is poor. A study conducted in Uganda reported that about $34 \%$ of the studied population were not aware of health insurance. ${ }^{1}$ Whereas, proportion of people having inadequate knowledge about health insurance was found to be high in countries such as India $(46 \%),{ }^{5}$ Myanmar $(66 \%)^{3}$ and Hispanic American in the USA $(70 \%) .{ }^{19}$

Familiarity or awareness of the insurance schemes increases the utilisation of health insurance and subsequently help in healthcare uptake. ${ }^{12}{ }^{19}$ Individuals usually enrol into health insurance because of their personal experiences, awareness or word-of-mouth advertisements. ${ }^{17}$ Mass media such as newspaper, radio and television play an important role in making people aware of health insurance schemes. ${ }^{1}$ Friends, community meetings, school gatherings and health workers have an influence on increasing the health insurance awareness of the people. ${ }^{1}$ Although, aforesaid factors help in increasing the awareness and enrolment in health insurance scheme, some enrollees may not pay premium on regular basis and might not get to know even after health insurance is lapsed. ${ }^{9}$ Women farmers, as compared with other occupations, had more odds of unawareness that their insurance was lapsed. ${ }^{9}$ 
It is evident from the above description that there is inadequate awareness of health insurance among general population in LMICs. Knowledge about health insurance can boost individuals' confidence and self-efficacy; thus, it is an important a priori factor that is required to get enrolled in health insurance scheme. ${ }^{19}$ Outreach programmes to increase general knowledge of health insurance and integrating health insurance education within health delivery systems may help to improve the uptake of health insurance. ${ }^{19}$ Globally, there are different methods available to promote and raise awareness about different health insurance schemes. However, India is a diverse country with a complex health system and numerous contextual factors. A 'one size fits all' approach for any policy or intervention is not suitable for the country. Therefore, it is imperative to understand the different approaches implemented to raise awareness about health insurance in the country. Additionally, due to increased population and a meagre public health spending on healthcare, it is important to understand if the resources are being used appropriately. To ensure this, understanding the effectiveness of such policies is essential, so that focus is directed towards the suitable interventions. 'Ayushman Bharat Scheme-PMJAY' is implemented to ensure increased utilisation of the healthcare facilities with financial protection of the beneficiaries. The evidence available on the effectiveness of the PMJAY scheme states no effect of the scheme on utilisation of healthcare and financial protection of enrolled beneficiaries ${ }^{13}$ however, this evidence is limited. Therefore, in the Indian context, it is important to understand if awareness is a factor that has led to decreased utilisation of PMJAY or failure of the other schemes (viz. Rashtriya Swasthya Bima Yojana-RSBY) in securing financial protection of the beneficiaries. ${ }^{20}$ Also, it is vital to understand the importance of awareness programmes for success of the health insurance schemes, which will be the focus of this review.

A systematic review will help in synthesising high-quality evidence in a systematic manner, for this important topic of interest. The proposed systematic review will, therefore, identify the different approaches and interventions for increasing health insurance awareness in India and will give information about the impact of these interventions. This review is planned to address the following research questions:

1. What are the various interventions implemented in India to promote awareness of health insurance?

2. What is the effectiveness of the above interventions on the awareness and uptake of health insurance by people of India?

\section{METHODS AND ANALYSIS}

Methodology for this systematic review will be based on the Cochrane handbook for systematic reviews of interventions $^{21}$ and we have adhered to Preferred Reporting Items for Systematic Reviews and Meta-Analyses
(PRISMA)-Protocols guidelines for reporting this protocol. $^{22}$

\section{Criteria for including studies in the review}

Population: The review will include studies conducted in India that involve adult population ( $>18$ years). We will consider the studies having household as a unit of analysis, if the head of the family (or the family member who was interviewed) is an adult.

Intervention/exposure: We will include any intervention, policy or programme that directly or indirectly affects awareness of health insurance. The health insurance scheme could be of any type, including but not limited to, public, private, for profit and not-for-profit. Contribution for premiums could be made by individual, NGO, employer or government. There is no restriction on focus of health insurance for example, hospital stay, surgery or critical illness.

Intervention/exposure could be educational, informative, training, technology and m-health or e-health related. The interventions could be focused on raising income threshold to be eligible for health insurance, such as, conditional or unconditional cash transfers that indirectly influences awareness of health insurance. Similarly, training and performance-based financing for healthcare staff or other groups will be eligible for inclusion. The intervention could be a modification of the enrolment procedure, changes in the premium or organisational changes in handling health insurance. Intervention could be directed on general population or targeted groups such as vulnerable population, indigenous groups, community leaders, employees, formal or informal groups and healthcare staff.

Comparison: This review will not restrict the studies based on comparison, as having a comparison group may not always be feasible.

Outcomes:

1. Awareness/health insurance literacy (refers to knowledge of the household head or household member on the presence of insurance schemes, its principles and significance. The outcome measure can be objective or subjective).

2. Attitude: Readiness to buy health insurance, decision making.

3. Uptake of health insurance.

4. Demand-side and supply-side factors for awareness of health insurance.

5. Awareness of health insurance as a factor for uptake or re-enrolment of health insurance.

Types of study designs: This review will include experimental studies that assessed the effect of intervention to promote awareness and uptake of health insurance. It is sometimes not practical to conduct randomised controlled trials (RCTs) to measure the effect of public health interventions, therefore, the review will also include other study designs. Studies with following designs will be included: RCTs, interrupted time-series studies, difference-in-difference, regression discontinuity 
designs, statistical matching, quasi-randomised and nonrandomised trials. Additionally, this review will include prospective, retrospective, analytical cross-sectional and studies related to process evaluation and policy analysis, if the studies have provided description of intervention or exposure of our interest. Qualitative studies are important source of information about barriers and enabling factors that can complement the findings, therefore, we will also include these types of studies. This review will exclude descriptive cross-sectional (prevalence) studies, commentaries, perspectives, editorials, reviews and conference abstracts. Policy papers that do not provide details of implementation of intervention will not be considered.

\section{Searching and locating the studies}

The electronic databases such as MEDLINE (PubMed), Web of Science and Scopus will be searched to identify potential records. Additionally, 3ie impact evaluation repository and Social Science Research Network will be searched. Databases will be searched from January 2010 to 15 July 2020 and publications will be restricted to English language. Ministry of Health and Family Welfare, RSBY, Ayushman Bharat and other state health insurance websites will be searched for reports on the health insurance schemes. We will also scan through references of the included studies for any additional eligible records. After identifying the keywords, initial search will be carried out in PubMed, which will then be replicated in other databases. A designated information scientist will be responsible for conducting search. The preliminary search concepts and key terms are given in table 1 .

\section{Applying eligibility and screening the studies}

The results of search will be imported to Endnote X7 reference manager software and duplicates will be removed.
MS Excel spreadsheet will be used to screen the records. Based on inclusion and exclusion criteria, all the records will be subjected to two stage-title/abstracand full text (FT) - screening process, independently by three (SSP, ER and BTV) reviewers (in pairs). Any disagreements between the reviewers will be resolved by discussion, and senior reviewer will be involved in decision making in case of disagreements between the reviewers. The reasons for excluding FTs will be documented and the PRISMA flow diagram will be provided. A detailed screening protocol will be used as a back-up document to aid the screening process. Table 2 gives detailed screening protocol.

\section{Data extraction}

Data will be extracted independently by three reviewers (SSP, ER and BTV). A predesigned data extraction form will be used for extraction of the data. The data extraction form will be subjected to pilot testing and will be revised as per the suggestions by the reviewers and the experts at this stage. Any disagreements during data extraction will be resolved by consensus supported by the senior reviewer. Data will be extracted based on the characteristics mentioned in the table 3 .

\section{Critical appraisal of included studies}

Effective Public Health Practice Project (EPHPP) tool ${ }^{23}$ will be used to assess the methodological quality of quantitative studies (except observational studies) and Newcastle-Ottawa scale (NOS) ${ }^{24}$ will be used for the observational studies. The EPHPP rates the study as 'strong', 'moderate' or 'weak' based on eight domains. These domains are selection bias, study design, confounders, blinding, data collection methods, withdrawals and dropouts, intervention integrity and analysis. ${ }^{23}$ NOS rates the study based on three domains viz. selection, comparability

Table 1 Search concepts and key words

\begin{tabular}{|c|c|}
\hline Concept & Key terms \\
\hline $\begin{array}{l}\text { Intervention or } \\
\text { exposure }\end{array}$ & $\begin{array}{l}\text { 'Information Education Communication', 'Mass Media', 'Television', 'Leaflet', 'Brochure', 'Flyer', } \\
\text { 'Radio', 'Television', 'Advertisement', 'Behavioural change communication', Awareness programme/ } \\
\text { programme/ campaign/ initiatives/policy', 'Promotion', 'Marketing', 'Social media', 'E-health', 'M- } \\
\text { health'. }\end{array}$ \\
\hline Insurance names & $\begin{array}{l}\text { 'Community insurance', 'Health insurance', 'Health insurance programmes/programmes/schemes', } \\
\text { 'Health finance /financing', 'Healthcare reform', 'Insurance coverage', 'National health insurance', } \\
\text { 'National health insurance scheme', 'Medical insurance', 'Micro health insurance', 'Public health } \\
\text { insurance', 'Social insurance', 'Social protection', 'Universal healthcare', 'Universal healthcare', } \\
\text { 'Ayushman Bharat', 'Pradhan Mantri Jan Arogya Yojana', 'Mukhyamantri Swasthya Bima Yojana', } \\
\text { 'Rashtriya Swastya Bima Yojana', 'Aarogysi', 'Rajiv Arogyabhagya', 'Rajiv Arogyashree health } \\
\text { insurance', 'Rajiv Gandhi Jeevandayee Arogya Yojana', 'Yesasvini health insurance', 'Yashshvini } \\
\text { Community based health insurance programme', 'Vajpayee Arogyashree', 'Biju Krushak Kalyan', } \\
\text { 'Kalainagar', 'CHIS', 'Employee State Insurance Scheme', 'Central Government Health Scheme', } \\
\text { 'Mediclaim', 'Deen Dayal Swasthya Seva Yojana'. }\end{array}$ \\
\hline Region & India \\
\hline
\end{tabular}




\section{Table 2 Screening protocol}

\section{$1 \quad$ Title and abstract screening}

A Is the study published in English? AND Is it If answer to both the components published in the year 2000 or later? are 'yes', Go to B

B Is it a study conducted in India?

Does study involve one of the following
design or analysis: RCTs, interrupted time
series studies, difference-in-difference,
regression discontinuity designs, statistical
matching, quasi-randomised and non-
randomised trials, prospective, retrospective
and analytical cross-sectional studies and
studies related to process evaluation and
policy analysis.

D Does the study describe the intervention for increasing awareness of and uptake of health insurance?

(The intervention could be any intervention, policy or programme (eg, behavioural change communication or educational) that directly or indirectly affects awareness of health insurance. There is no restriction on mode of intervention, for example, mass media or group discussions. There is no restriction on who provides the intervention, for example, researcher, community-based workers or insurance agent. There is no restriction on duration and frequency of providing intervention. The health insurance scheme could be of any type, including but not limited to, public, private, for profit and not-for-profit. Contribution for premiums could be made by individual, nongovernmental organisations, employer or government. There is no restriction on focus of health insurance, for example, hospital stay or surgery.)OR

Does the study describe about the factors associated with awareness of health insurance? OR Does the study describe awareness as a factor for uptake or reenrolment of health insurance?

\section{If answer to one of the} components is 'yes' OR if it is not clearly stated and you are in doubt, then Include the study for full-text screening

If you are in doubt: flag for discussion
If it is non-English or published before 2000 then exclude the study

1. If it is 'yes' OR

If it is clearly stated that it is conducted elsewhere, but India, then exclude the study

If answer is 'yes' OR it is not clearly stated in abstract, Go to D

If the study is descriptive crosssectional (or prevalence study) having single group OR if the publication is a commentary, perspective, editorial, reviews, conference abstracts OR policy paper that does not provide details of implementation of intervention: exclude the study If no, exclude the study

\begin{tabular}{|c|c|c|c|}
\hline 2 & Full-text screening & & \\
\hline$E$ & Is it a study conducted in India? & If it is 'yes', Go to F & If no exclude the study \\
\hline $\mathrm{F}$ & Did the study involve adult population? & If it is 'yes', Go to G & If no exclude the study \\
\hline
\end{tabular}




\begin{tabular}{|c|c|c|c|}
\hline 1 & Title and abstract screening & & \\
\hline $\mathrm{H}$ & $\begin{array}{l}\text { Does the study describe the intervention } \\
\text { for increasing awareness of and uptake of } \\
\text { health insurance? } \\
\text { (The intervention could be any intervention, } \\
\text { policy or programme (eg, behavioural } \\
\text { change communication or educational) that } \\
\text { directly or indirectly affects awareness of } \\
\text { health insurance. } \\
\text { There is no restriction on mode of } \\
\text { intervention, for example, mass media or } \\
\text { group discussions. There is no restriction on } \\
\text { who provides the intervention, for example, } \\
\text { researcher, community-based workers or } \\
\text { insurance agent. There is no restriction } \\
\text { on duration and frequency of providing } \\
\text { intervention. The health insurance scheme } \\
\text { could be of any type, including but not } \\
\text { limited to, public, private, for profit and not- } \\
\text { for-profit. Contribution for premiums could } \\
\text { be made by individual, non-governmental } \\
\text { organisations, employer or government. } \\
\text { There is no restriction on focus of health } \\
\text { insurance, for example, hospital stay or } \\
\text { surgery.) OR } \\
\text { Does the study describe about the factors } \\
\text { associated with awareness of health } \\
\text { insurance? } \\
\text { OR Does the study describe awareness as } \\
\text { a factor for uptake or re-enrolment of health } \\
\text { insurance? }\end{array}$ & $\begin{array}{l}\text { If answer to one of the } \\
\text { components is 'yes' Go to I OR } \\
\text { If you are in doubt, then flag for } \\
\text { discussion }\end{array}$ & If no exclude the study \\
\hline
\end{tabular}

$\mathrm{RCT}$, randomised controlled trial.

and outcome, and the final score ranges between 0 and $10 .{ }^{24}$ Reviewers (BTV, ER and SSP), independently in pairs, will appraise the included studies. Any discrepancies between the decisions of reviewers will be resolved by discussion until consensus is achieved. If required, a senior reviewer will be involved as arbitrator and final decision maker to rate the study quality.

\section{Data analysis}

Study characteristics consisting of population, intervention/exposure, comparator, outcome, study design components across studies will be tabulated, which will help us to compare and analyse. Subsequently, studies will be categorised into quantitative and qualitative and will be analysed separately. This step will be followed by mixed methods synthesis as suggested by Panda et al. ${ }^{25}$

\section{Quantitative studies}

Studies will be grouped based on study design, and type of data available (continuous or categorical). If possible, similar studies will be pooled to perform meta-analysis using random effect model. If data are continuous, standardised mean difference will be calculated with $95 \%$ CI.
For categorical data, OR or risk ratio will be calculated and reported with $95 \%$ CI. Meta-analysis will be visually represented with a forest plot. We assume possibility of heterogeneity owing to differences in study design or analysis, intervention, type of insurance and other contextual factors. If heterogeneity exists due to aforementioned components, we will not perform meta-analysis. After ruling out clinical or methodological heterogeneity, we will statistically measure heterogeneity by using $\mathrm{I}^{2}$ test. If significant heterogeneity $(>50 \%)$ persists for a particular outcome, meta-analysis will not be conducted. In this case, our focus would be on conducting narrative synthesis and undertaking a subgroup analysis. Key findings of the studies will be summarised in tables/figures or vote counting will be considered. Subgroups could be based on study design, intervention type, insurance type (such as private and public), region and other contextual factors (eg, urban/rural).

\section{Qualitative synthesis}

We will carry out thematic analysis as suggested by Thomas and Harden. ${ }^{26}$ An iterative process of line-by-line coding 
Table 3 Data extraction format

\begin{tabular}{|c|c|}
\hline \multirow[b]{3}{*}{ Publication details } & First author's last name \\
\hline & Year of publication \\
\hline & Publication type: report/ journal publication \\
\hline \multirow{2}{*}{$\begin{array}{l}\text { Population } \\
\text { characteristics }\end{array}$} & Gender \\
\hline & Religion/race/ethnicity \\
\hline \multirow[t]{2}{*}{ Location/setting } & State/district or other details of place where study was conducted \\
\hline & $\begin{array}{l}\text { Setting: hospital/community based } \\
\text { Rural/urban }\end{array}$ \\
\hline $\begin{array}{l}\text { Study methodology/ } \\
\text { design }\end{array}$ & $\begin{array}{l}\text { Study design: RCT, quasi-randomised trial, case-control study. } \\
\text { Type of analysis }\end{array}$ \\
\hline Insurance details & $\begin{array}{l}\text { Public/private/community-based insurance } \\
\text { Start or launch date (month and year) of insurance } \\
\text { Type of plan, for example, individual, family, senior citizen, critical illness. } \\
\text { Benefits of health insurance, for example, cashless facility, hospitalisation, prehospitalisation and } \\
\text { posthospitalisation, medical check-up, maternity benefits, childcare, critical illness. }\end{array}$ \\
\hline Exposure details & List different factors or themes \\
\hline \multirow[t]{2}{*}{ Outcome details } & $\begin{array}{l}\text { List down outcome, variable type: continuous or categorical, type of analysis } \\
\text { Effect measures with } 95 \% \mathrm{Cl} \text { (such as OR, risk ratio, HR) } \\
\text { No of participants analysed, number lost to follow-up } \\
\text { Details of subgroup analysis, if any. }\end{array}$ \\
\hline & Themes and subthemes \\
\hline
\end{tabular}

Other details

$\mathrm{RCT}$, randomised controlled trial.

will be undertaken as a first step, which will be followed by categorising the codes into code families. Subsequently, a code tree will be created, and themes and subthemes will be generated. Three reviewers (SSP, ER and BTV) will code the data independently and resolve the discrepancies by discussion until consensus is achieved.

\section{Mixed-methods synthesis}

The result from both, qualitative and quantitative synthesis will be merged for each outcome. Parallel synthesis will be carried out, and the findings will be summarised narratively. ${ }^{25}$ To understand the influence of inequality in uptake of health insurance based on type of insurance, we will explore the possibility of conducting subgroup analysis based on some of the components of PROGRESS (Place of residence, Race/ethnicty/culture/ language, Occupation, Gender, Religion, Education, Socio-economic status, Social capital)-Plus framework. ${ }^{27}$

\section{Grading the evidence}

We will use the Grading of Recommendations Assessment, Development and Evaluation (GRADE) approach to evaluate the certainty of evidence for each outcome. ${ }^{21}$ Using GRADE profiler software, we will present the main findings of the systematic review in a summary of findings table.

\section{Patient and public involvement}

We did not involve patients or public while designing and writing this protocol.

\section{Ethics and dissemination}

This review will be based on published studies, therefore, an ethical clearance is not applicable. We have planned following activities to communicate and disseminate the findings of this review. We plan to make at least one national or international conference presentation. We will prepare policy brief to be shared with funder and to get a wider reader, we plan to submit the manuscript to a peer-reviewed journal. On journal publication, we intend to circulate the findings through our social media platform and website.

\section{Twitter Shradha S Parsekar @ParsekarShrads}

Acknowledgements We are grateful to Dr Prachi Pundir, Research Officer, public Health Evidence South Asia, Prasanna School of Public Health, Manipal Academy of Higher Education, Manipal, for proof reading the final document. 
Contributors RB is the guarantor of the review. RB, SSP, ER and BTV conceptualised the manuscript. SSP drafted the first manuscript, which was further edited by ER. RV developed the search strategy. All the authors (RB, BU, SSP, ER, RV and BTV) read, edited, provided feedback and approved the final manuscript.

Funding This work was supported by PHRI-RESEARCH grants awarded by PHFI with the financial support of Department of Science and Technology (DST). We appreciate the technical support provided by public Health Evidence South Asia, Prasanna School of Public Health, Manipal Academy of Higher Education, Manipal.

Disclaimer Funder did not have any role in writing this protocol and decision to submit it for publication.

Competing interests None declared.

Patient and public involvement Patients and/or the public were not involved in the design, or conduct, or reporting, or dissemination plans of this research.

Patient consent for publication Not required.

Provenance and peer review Not commissioned; externally peer reviewed.

Open access This is an open access article distributed in accordance with the Creative Commons Attribution Non Commercial (CC BY-NC 4.0) license, which permits others to distribute, remix, adapt, build upon this work non-commercially, and license their derivative works on different terms, provided the original work is properly cited, appropriate credit is given, any changes made indicated, and the use is non-commercial. See: http://creativecommons.org/licenses/by-nc/4.0/.

ORCID iDs

Shradha S Parsekar http://orcid.org/0000-0002-8824-9198

Bhumika Tumkur Venkatesh http://orcid.org/0000-0002-3338-6478

\section{REFERENCES}

1 Basaza R, Kyasiimire EP, Namyalo PK, et al. Willingness to pay for community health insurance among TAXI drivers in Kampala City, Uganda: a contingent evaluation. Risk Manag Healthc Policy 2019;12:133-43.

2 Mirach TH, Demissie GD, Biks GA. Determinants of communitybased health insurance implementation in West Gojjam zone, Northwest Ethiopia: a community based cross sectional study design. BMC Health Serv Res 2019;19:544.

3 Myint C-Y, Pavlova M, Groot W. Health insurance in Myanmar: knowledge, perceptions, and preferences of social security scheme members and general adult population. Int $J$ Health Plann Manage 2019;34:346-69.

4 Ogundeji YK, Akomolafe B, Ohiri K, et al. Factors influencing willingness and ability to pay for social health insurance in Nigeria. PLoS One 2019;14:e0220558-e58.

5 Surendar RAM, Madhumadhi S, Saravanan R. Awareness on health insurance among a selected urban population in Puducherry. Nat $J$ Res Community Med 2019;8:176-9.

6 Okoroh J, Essoun S, Seddoh A, et al. Evaluating the impact of the National health insurance scheme of Ghana on out of pocket expenditures: a systematic review. BMC Health Serv Res 2018;18:426.

7 Kuwawenaruwa A, Ramsey K, Binyaruka P, et al. Implementation and effectiveness of free health insurance for the poor pregnant women in Tanzania: a mixed methods evaluation. Soc Sci Med 2019;225:17-25.

8 van Hees SGM, O'Fallon T, Hofker M, et al. Leaving no one behind? social inclusion of health insurance in low- and middle-income countries: a systematic review. Int J Equity Health 2019;18:134.

9 Kanmiki EW, Bawah AA, Akazili J, et al. Unawareness of health insurance expiration status among women of reproductive age in northern Ghana: implications for achieving universal health coverage. $\checkmark$ Health Popul Nutr 2019;38:34.
10 Fadlallah R, El-Jardali F, Hemadi N, et al. Barriers and facilitators to implementation, uptake and sustainability of community-based health insurance schemes in low- and middle-income countries: a systematic review. Int J Equity Health 2018;17:13.

11 Maurya D, Mintrom M. Policy entrepreneurs as catalysts of broad system change: the case of social health insurance adoption in India. $J$ Asian Public Policy 2020;13:18-34.

12 Lagomarsino G, Garabrant A, Adyas A, et al. Moving towards universal health coverage: health insurance reforms in nine developing countries in Africa and Asia. Lancet 2012;380:933-43.

13 Garg S, Bebarta KK, Tripathi N. Performance of India's national publicly funded health insurance scheme, Pradhan Mantri Jan Arogaya Yojana (PMJAY), in improving access and financial protection for hospital care: findings from household surveys in Chhattisgarh state. BMC Public Health 2020;20:949.

14 NSS 75th Round. Key indicators of social consumption in India health. New Delhi The government of India, MInistry of Statistics and Programme Implementation, National Statistical Office; 2019. http:// mospi.nic.in/sites/default/files/publication_reports/KI_Health_75th_ Final.pdf

15 Abiola AO, Ladi-Akinyemi TW, Oyeleye OA, et al. Knowledge and utilisation of national health insurance scheme among adult patients attending a tertiary health facility in Lagos state, south-western Nigeria. Afr J Prim Health Care Fam Med 2019;11:e1-7.

16 Seddoh A, Sataru F. Mundane? demographic characteristics as predictors of enrolment onto the National health insurance scheme in two districts of Ghana. BMC Health Serv Res 2018;18:330.

17 Michael GC, Aliyu I, Grema BA, et al. Perception of factors influencing primary health-care facility choice among National health insurance enrollees of a northwest Nigerian Hospital. J Patient Exp 2019;6:247-52

18 Ayanore MA, Pavlova M, Kugbey N, et al. Health insurance coverage, type of payment for health insurance, and reasons for not being insured under the National health insurance scheme in Ghana. Health Econ Rev 2019;9:39.

19 Ghaddar S, Byun J, Krishnaswami J. Health insurance literacy and awareness of the Affordable care act in a vulnerable Hispanic population. Patient Educ Couns 2018;101:2233-40.

20 Prinja S, Chauhan AS, Karan A, et al. Impact of publicly financed health insurance schemes on healthcare utilization and financial risk protection in India: a systematic review. PLoS One 2017;12:e017 0996-e96.

21 Higgins JPT, Thomas J, Chandler J, et al, eds. Cochrane Handbook for systematic reviews of interventions version 6.1 (updated September 2020). London: Cochrane, 2020.

22 Moher D, Liberati A, Tetzlaff J, et al. Preferred reporting items for systematic reviews and meta-analyses: the PRISMA statement. PLoS Med 2009;6:e1000097.

23 Effective Public Health Practice Project. Quality assessment tool for quantitative studies. Hamilton, ON: Effective Public Health Practice Project, 1998. https://www.nccmt.ca/knowledge-repositories/search/ 14

24 Wells GA, Shea B, O'Connell D. The Newcastle-Ottawa scale (NOS) for assessing the quality of nonrandomised studies in meta-analyses. Ottawa: Ottawa Hospital Research Institute, 2019. http://www.ohri. $\mathrm{ca/programs/clinical \_ epidemiology/oxford.asp}$

25 Panda P, Dror I, Perez Koehlmoos T, et al. What factors affect take up of voluntary and community based health insurance programmes in low- and middle- income countries? A systematic review (protocol. London: EPPI-Centre, Social Science Research Unit, Institute of Education, University of London, 2013.

26 Thomas J, Harden A. Methods for the thematic synthesis of qualitative research in systematic reviews. BMC Med Res Methodol 2008;8:45.

27 Oliver S, Kavanagh J, Caird J. Health promotion, inequalities and young people's health. A systematic review of research. London: EPPI-Centre, Social Science Research Unit, Institute of Education, University of London, 2008. 